\title{
Key performance requirement of future next wireless networks
} (6G)

\author{
Ahmad A. A. Solyman', Khalid Yahya ${ }^{2}$ \\ ${ }^{1}$ Department of Electrical and Electronics Engineering, Faculty of Engineering and Architecture, Istanbul Gelisim \\ University, Turkey \\ ${ }^{2}$ Department of Mechatronic Engineering, Faculty of Engineering and Architecture, Istanbul Gelisim University, Istanbul, \\ Turkey
}

\begin{tabular}{|c|c|}
\hline Article Info & ABSTRACT \\
\hline Article history: & Given the massive potentials of $5 \mathrm{G}$ communication networks and their \\
\hline Received Sep 19, 2020 & $\begin{array}{l}\text { foreseeable evolution, what should there be in } 6 \mathrm{G} \text { that is not in } 5 \mathrm{G} \text { or its long- } \\
\text { term evolution? } 6 \mathrm{G} \text { communication networks are estimated to integrate the }\end{array}$ \\
\hline Revised May 4, 2021 & terrestrial, aerial, and maritime communications into a forceful network which \\
\hline Accepted Oct 1, 2021 & $\begin{array}{l}\text { would be faster, more reliable, and can support a massive number of devices } \\
\text { with ultra-low latency requirements. This article presents a complete overview }\end{array}$ \\
\hline Keywords: & $\begin{array}{l}\text { of potential } 6 \mathrm{G} \text { communication networks. The major contribution of this study } \\
\text { is to present a broad overview of key performance indicators (KPIs) of } 6 \mathrm{G}\end{array}$ \\
\hline $\begin{array}{l}6 \mathrm{G} \text { communications } \\
\text { Key performance indicators }\end{array}$ & $\begin{array}{l}\text { networks that cover the latest manufacturing progress in the environment of } \\
\text { the principal areas of research application, and challenges. }\end{array}$ \\
\hline
\end{tabular}

This is an open access article under the $\underline{C C B Y-S A}$ license.

Wireless networks

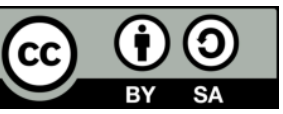

\section{Corresponding Author:}

Ahmad A. A. Solyman

Department of Electrical and Electronics Engineering

Faculty of Engineering and Architecture

Istanbul Gelisim University, Turkey

Email: aaasahmed@gelisim.edu.tr

\section{INTRODUCTION}

Figure 1 summarizes the major milestones of the five generations (1G-5G) of wireless communication networks. 5G communications have been standardized and are already being used all over the world [1]. ITU defines the vision and requirements; $5 \mathrm{G}$ would get together eight key performance indicators (KPIs) and three common scenarios [2]. The three challenging technical indicators for all three scenarios are the millions of interconnects per square kilometer $\left(1 \mathrm{M} / \mathrm{km}^{2}\right)$ of huge machine-type communications, the millisecond (ms) air interface delay of ultra-reliable low-latency communications (URLLC), and millions of square kilometers (ms) of interconnection. Enhanced mobile broadband (eMBB) (mMTC). A collection of enabling technologies was proposed argued in standardizations, and tested in technological trials to achieve these KPIs [3]. Massive MIMO, advanced coding and modulation, mmWave communication, non-orthogonal multiple access, ultra-dense networking (UDN), dual connection architecture, and adjustable frame structure are only a few examples of wireless technology [4], [5].

However, because of the significant increase in the number of interconnected devices, which could reach hundreds of devices per cubic meter, a tremendous increase in data exchange is expected; Apart from the growing number of new applications like virtual/augmented reality (VR/AR), self-driving cars, threedimensional integrated communications, and yet-to-be-conceived applications [4], [6], 5G networks would require even higher data rates and lower latency. These constraints are seen as a key driver in the 
development of $6 \mathrm{G}$ communication technologies. What could be better in $6 \mathrm{G}$ that is not included in $5 \mathrm{G}$, given the vast potentials of $5 \mathrm{G}$ networks and their anticipated evolution? Academic, industry, and research organizations are busy describing and identifying important essential enabling technologies that could characterize $6 \mathrm{G}$, with deployment estimated by 2030 [7], [8].

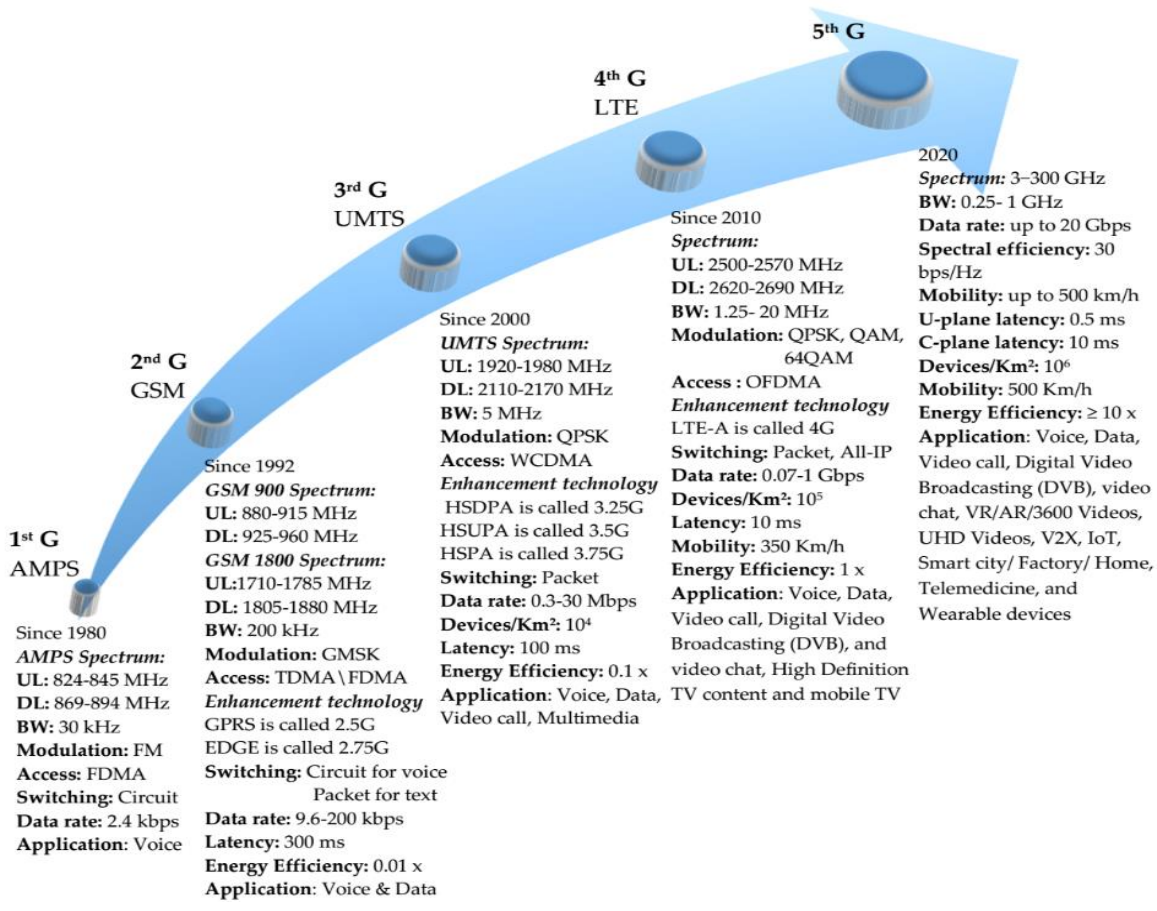

Figure 1. Different generations of communications (1G-5G) Major milestones

Based on the vision and expansion of a universal smart Mobile Sphere, 6G will be improved and broadened to allow for a 10 to 100-fold increase in data throughput, increased system capacity, wider and deeper coverage, higher resource utilization, higher spectrum efficiency, support higher moving speeds, lower delay, and entirely support the progress of a universal smart Mobile Sphere. The vision's expected requirements will be discussed in great detail in the following paragraphs:

- $6 \mathrm{G}$ ought to be an omnipresent and well-connected system with larger and wider coverage, incorporating terrestrial, short-range device-to-device communication, and satellite, among other things. 6G can operate in a variety of situations, including airspace, land, and sea, thanks to sophisticated mobility management technologies, resulting in a universal, inescapable mobile broadband communication system.

- $6 \mathrm{G}$ is a network that is extremely tailored and intelligent. The combination of $6 \mathrm{G}$ and artificial intelligence makes personal mobile communications virtual, and the network is transformed from the traditional centralized function to the new 3-centralized type of centralized user-centric, data-centric and full-content services.

- $6 \mathrm{G}$ is estimated to run at upper frequencies, like $4 \times 10^{14}$ to $8 \times 10^{14} \mathrm{~Hz}$ (visible light), higher frequency radio bands ranging from $24 \mathrm{GHz}$ to $40 \mathrm{GHz}$ (mmWave), frequencies between $100 \mathrm{GHz}$ and $10 \mathrm{THz}$ (terahertz), and so on, in order to achieve a wider bandwidth. When compared to $5 \mathrm{G}$, $6 \mathrm{G}$ can increase data rates by up to 100 times, allowing for peak data rates of $\mathrm{Tb} / \mathrm{s}$ and user data rates of $10 \mathrm{~Gb} / \mathrm{s}$. Furthermore, 6G can make use of the reconfigurable frequency sharing technique to increase the efficiency of frequency reuse even further.

- The 6G network will adopt an endogenous safety system or an integrated functional safety design. By combining trust and security procedures, 6G can achieve self-awareness, dynamic real-time analysis, flexible risk, and trust assessment, all of which will contribute to cyberspace security.

- 6G communications will include computing, navigation, and detection. Satellite communication systems, satellite navigation and positioning systems, and perhaps radar detection systems, will all become part of 6G. 6G will have a more open architecture with a software-defined core network and wireless access network, allowing faster and more conscious development and dynamic deployment of network operations. 
- Because of the internet of things (IoT), 6G can generate massive amounts of data; It can also be combined with new technologies, such as artificial intelligence, edge computing, cloud computing, and blockchain. 6G can support the ubiquitous intelligent mobile society by realizing everything related to intelligence and collective intelligence (swarm intelligence).

This paper intends to highlight the significant concerns of key performance indicators (KPIs) of $6 \mathrm{G}$ networks that are not covered by 5G, as well as the most current developments in the sector in terms of the main application areas and challenges. To that purpose, our research attempted to take as many approaches as workable. Due to space constraints, this work examined disputed research topics in-depth based on their subdomains in order to get a precise and concise conclusion. This publication will open up new research possibilities for scholars by providing various new references that could aid in the goal of creating 6G networks. The rest of the article is structured as follows. The primary KPIs of $6 \mathrm{G}$ wireless systems discussed in section 2 include max data rate, needs for mobility, connected devices $/ \mathrm{Km}^{2}$, dependability, area traffic capacity, latency, network spectrum, and energy efficiency, followed by a comparison of KPIs between 5G and $6 \mathrm{G}$ networks. The work comes to a close in section 3.

\section{KEY PERFORMANCE INDICATORS OF 6G NETWORKS}

The prime KPIs of $6 \mathrm{G}$ wireless systems are discussed in this section. Figure 2 shows the key performance indicators (KPIs) for max data rate, dependability, needs for mobility, connected devices $/ \mathrm{Km}^{2}$, area traffic capacity, latency, network spectrum, and efficiency in terms of energy. In addition, Figure 3 shows a comparison of KPIs across $5 \mathrm{G}$ and $6 \mathrm{G}$ networks.



Figure 2. The main KPIs of $6 \mathrm{G}$ wireless networks

\subsection{Data rate}

Since the inception of wireless communications, users' data rate requirements have risen. The data speeds in $1 \mathrm{G}$ were a small number of kbps, which grew to $10^{6} \mathrm{bps}$ in $5 \mathrm{G}$. These data rates are insufficient for several applications. As a result, $6 \mathrm{G}$ is planned to have more bandwidth by operating at a higher frequency, such as visible light and Terahertz. 6G can boost data rates by 10 to 100 times compared to $5 \mathrm{G}$, allowing for Tbps peak data rates. The general view is that data rates would increase to $1 \mathrm{Tbps}$ in the future smart city to enable autonomous management of many operations. Individual data rates are predicted to rise from $1 \mathrm{Gbps}$ in $5 \mathrm{G}$ to at least $10 \mathrm{Gbps}$ for every user in upcoming $6 \mathrm{G}$ systems, and up to $100 \mathrm{Gbps}$ in specific use cases [9]. 6G can take advantage of flexible frequency sharing technologies to improve frequency reuse efficiency even more. As a result, we may download HD videos in a matter of seconds [10]. 


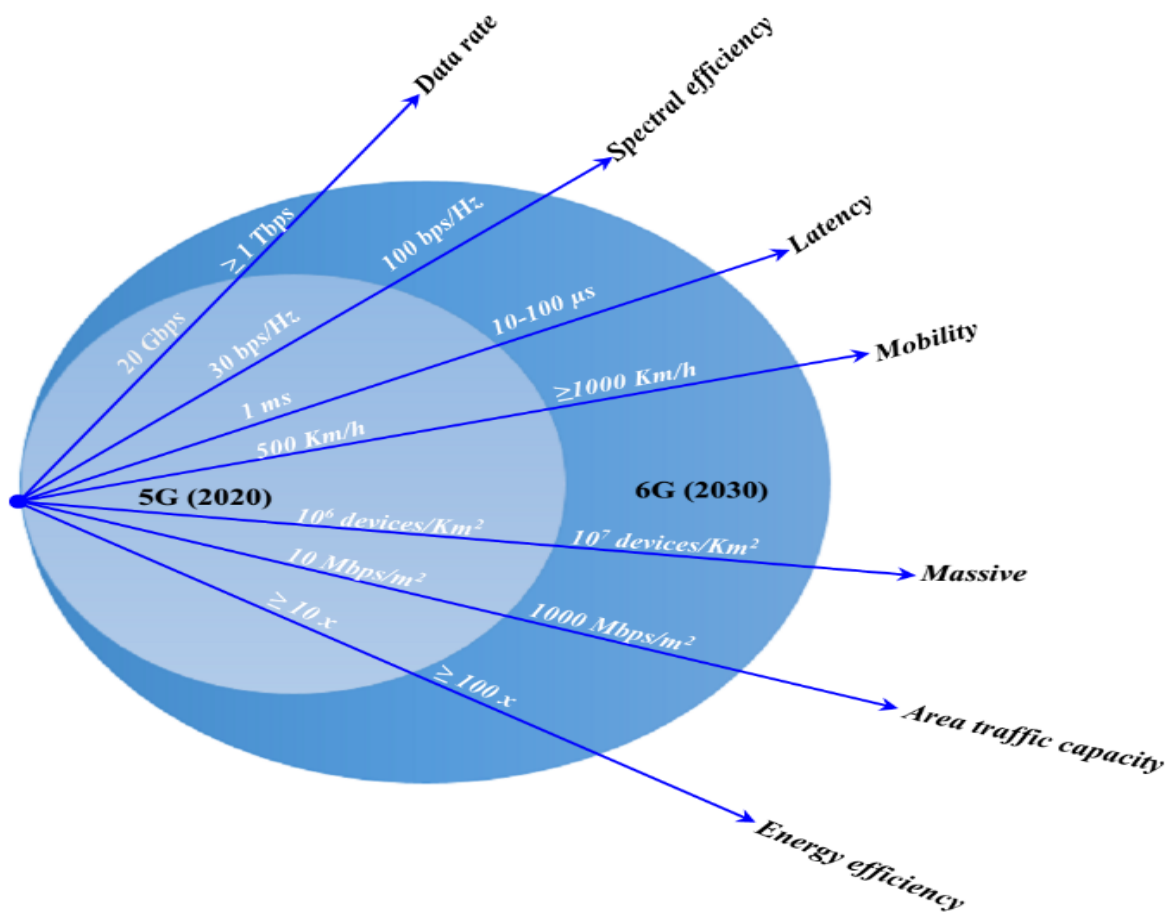

Figure 3 . KPIs for $5 \mathrm{G}$ versus $6 \mathrm{G}$

\subsection{Spectral efficiency}

Smart buildings will be employed in $6 \mathrm{G}$ to give another degree of freedom (DoF) to wireless networks, allowing them to deliver unprecedented capacity. On a large scale, buildings will be installed with smart reflecting surfaces [11]. The smart surfaces would effectively enlarge the antenna aperture to gather as many previously unavailable radio signals as it can for better energy and spectrum efficiency. Furthermore, employing polarization diversity and orbital angular momentum (OAM) mode multiplexing, it has been shown that a large capacity wireless communication system can be developed to operate within a few meters. Several independent data streams can be broadcast over the same spatial wireless channel, resulting in a tenfold increase in area spectral efficiency. The performance over a short distance is particularly encouraging, which could be valuable in automating several industries. According to reports, the millimeterwave OAM system in [12] has achieved a rate exceeding 2.5 Tbps and has a huge spectral efficiency of $95.7 \mathrm{bps} / \mathrm{Hz}$. This may be a profitable technology for Industry 4.0 and is expected to become one of the most important $6 \mathrm{G}$ use cases. In contrast, $6 \mathrm{G}$ is expected to work in conjunction with satellites to provide worldwide mobile coverage. In $6 \mathrm{G}$, volume spectral efficiency (in bps $/ \mathrm{Hz} / \mathrm{m} 3$ ) will be more appropriate for measuring system capability in a three-dimensional working space than the commonly employed area spectral efficiency (bps/Hz/m2).

\subsection{Latency}

The term "low latency" refers to communication that is swift and efficient. We want our packets to be sent in a quick length of time with little processing delay. In 6G, the maximum permitted latency is 10 seconds [13]. High dependability and ultra-low latency will be required for the future network of intelligent mobiles and robotics. The cities of the future will have smart cars, smart homes, smart schools/universities, smart industries, and smart industries. bullet trains, Ships, airplanes, and unmanned aerial vehicles (UAVs) will all need to be connected to smart cities. defense, health care, surveillance, and monitoring are just a few of the key applications that will cause ultra-reliability and low latency [14].

High dependability and minimal latency are required for online gaming services. The ERLLCS security feature on the $6 \mathrm{G}$ wireless system will be integrated with the mMTC and uRLLC on the 5G, and the reliability standard exceeds $99.9999999 \%$ (Nine-Nine) [15]. Self-driving vehicles must be linked to one another, and the link between them must be very reliable, otherwise, lives may be lost in accidents. Many houses and other sensors will connect with one other in $6 \mathrm{G}$ networks, causing ultra-reliability to avoid any mishaps. 


\subsection{Mobility}

A number of heterogeneous radios will be supported in $6 \mathrm{G}$ devices. Users are connected to the network as a whole rather than to a single cell, allowing multi-connectivity techniques to extend cell borders. Without manual intervention or configuration, the device will be able to seamlessly transition between different heterogeneous links (such as sub6 GHz, millimeter wave, terahertz, or VLC), providing QoS guarantees that meet the most demanding mobility requirements envisaged by $6 \mathrm{G}$, such as Low latency even in ultra-high mobility scenarios (up to $1000 \mathrm{~km} / \mathrm{h}$ ) [13].

\subsection{Massive connectivity}

mMTC is additional usage for next-generation wireless communication. The IoT eliminates the need for human interaction using machine-to-machine communication. From one machine to another, calls, messages, and commands are sent. The acts are not carried out by humans. The machines, rather than humans, are the ones who converse with one another. Wireless networks in the next generation are anticipated to have a capacity of 107 devices $/ \mathrm{km}^{2}$ [15], [16].

Sensor networks and the IoT will cooperate with each other and connect to many base stations. Portable devices, control and monitoring equipment, autonomous vehicles, smart grids, industrial automation, and control equipment, and medical and health-related devices are just some of the projects and applications in this field. Peer-to-peer or cooperative multi-hop relay communication is possible between these devices. A network infrastructure design capable of supporting a variety of content-driven applications and networks is required for various applications or devices. Taking all these factors into account, the next generation of wireless networks will require a new optimization and planning method.

\subsection{Area traffic capacity}

As the number of connected devices per unit area increases, the demand for higher capacity channels and backhaul increases. On a daily basis, a densely distributed sensor network creates more than tera-bytes (TB) of data [17]. To handle the traffic, this data output requires a high-capacity backhauling line.

Wireless protocols in earlier wireless generations (1G-to-5G) were built for specific uses. With the rise of massive IoTs or mMTC, We will have to develop equipment that is both energy-efficient and costeffective. This massive IoT connectivity resulted in the development of vehicle-to-vehicle communication, commonly known as V2X. The vehicle must interact with other vehicles, pedestrians, and various other sensors(vehicle-to-infrastructure). All of these communications must be highly reliable, low latency, and secure. Another case in point is industrial automation, which involves a huge number of sensors communicating and producing large amounts of data. For $6 \mathrm{G}$, the minimum area traffic capacity limit is 1000 $\mathrm{Mbps} / \mathrm{m}^{2}[2]$.

\subsection{Energy efficiency}

Many expectations will be met and exceeded with $6 \mathrm{G}$, including the supply of great energy performance, particularly in the context of widespread IoT usage and an eco-system of numerous minute sensors. In addition, because of the smartphone's ability to handle complex multimedia signal processing and the ability to increase by leaps and bounds with the increase in power consumption, it is imperative to expand the battery charging capacity [18]. Low power consumption and longer battery life are two research areas of $6 \mathrm{G}$ to solve the daily charging challenges faced by most communication devices and improve communication needs. As a result, a thorough energy-saving wireless communication strategy will be required for 6G. A key objective of $6 \mathrm{G}$ communication is to use battery-free communications whenever possible, with a communication efficiency of 1 Pico-joule per bit [7]. 6G communications provide the benefits of high-power terahertz waves, allowing devices to transmit power beams in specific directions, and use MIMO antenna arrays to guide beam communications. This method has the ability to offer enough power to devices inside the network's service area. According to the $6 \mathrm{G}$ vision and guidance outlined in [2], in $6 \mathrm{G}$, research should focus on battery life and service categories, rather than latency and data throughput. To save energy, the processing operations of the user nodes should be transferred to intelligent base stations with a reliable power supply or general intelligent radio space [19]. Cooperative relay communication and network densification are also crucial for reducing the single-hop signal propagation gap and mobile node transmission power [20], [21]. Long battery life in $6 \mathrm{G}$ is achieved through a mix of divergent energy-harvesting technologies that collect energy not only from surreal RF waves but similarly from sunlight and micro-vibrations [22], [23]. Wireless power charging over a long distance could also be a viable option for extending battery life [24], [25]. Furthermore, decentralized laser charging is a system that might give 2-W power to mobile devices safely over a distance of up to 10 meters [1].

Finally, we can say that the real breakthrough will come when considering frequencies beyond 100 GHz. The large bandwidth available here is of particular interest, first in the D-band at about $140 \mathrm{GHz}$ and then in the $300 \mathrm{GHz}$ range. Of course, they still exist. Some major challenges require technology solutions 
that can break the limits of silicon. Potential approaches also include hybrid silicon and IIIV technologies, aiming to achieve the $\mathrm{F}_{\max }=1 \mathrm{THz}$ transistor and very small antenna cointegration at the wafer or packet level. So far, the convergence of these solutions has not been found, but some trends are emerging. The CEALeti team is considering two specific aspects. In terms of transceivers, to avoid high bandwidth conversion and power consumption, channel bonding/aggregation has attracted people's attention and the $100 \mathrm{Gbps}$ performance of more than 16 channels has been proven. In terms of antennas, the focus of the work is to use a single transceiver/FEM transmission set, rather than the more classical phase shift architecture, which requires one transceiver/FEM per mosaic.

\section{CONCLUSION}

This research has taken a thorough and in-depth look at the fundamental key performance indicators that will be needed in the $6 \mathrm{G}$ era between 2025 and 2035. This study began by highlighting some of the most important aspects and implications of $6 \mathrm{G}$ wireless networks. The study then went over the key differences between $6 \mathrm{G}$ and $5 \mathrm{G}$ in terms of KPIs, such as max data rate, mobility needs, linked devices/Km2, area traffic capacity, latency, dependability, network spectrum, and energy efficiency. 6G will upgrade and expand 10100 times the data speed, larger system capacity, higher spectrum efficiency, lower latency, wider and deeper coverage, and fully empower the creation of a ubiquitous intelligent mobile society.

\section{ACKNOWLEDGEMENTS}

The author wishes to express his gratitude to the editors of BEEI as well as anonymous reviewers for their time and efforts in reviewing this manuscript.

\section{REFERENCES}

[1] M. H. Alsharif, A. H. Kelechi, M. A. Albreem, S. A. Chaudhry, M. S. Zia and S. Kim, "Sixth generation (6G) wireless networks: vision, research activities, challenges and potential solutions," Symmetry, vol. 12, p. 676, 2020, doi: 10.3390/sym12040676.

[2] K. David and H. Berndt, "6G vision and requirements: Is there any need for beyond 5G?," IEEE Vehicular Technology Magazine, vol. 13, no. 3, pp. 72-80, 2018, doi: 10.1109/MVT.2018.2848498.

[3] Z. Zhang et al., "6G wireless networks: Vision, requirements, architecture, and key technologies," IEEE Vehicular Technology Magazine, vol. 14, no. 3, pp. 28-41, 2019, doi: 10.1109/MVT.2019.2921208.

[4] S. Chen, Y.-C. Liang, S. Sun, S. Kang, W. Cheng and M. Peng, "Vision, requirements, and technology trend of 6G: how to tackle the challenges of system coverage, capacity, user data-rate and movement speed," IEEE Wireless Communications, vol. 27, no. 2, pp. 218-228, 2020, doi: 10.1109/MWC.001.1900333.

[5] M. Agiwal, A. Roy and N. Saxena, "Next generation 5G wireless networks: a comprehensive survey," IEEE Communications Surveys \& Tutorials, vol. 18, no. 3, pp. 1617-1655, thirdquarter 2016, doi: 10.1109/COMST.2016.2532458.

[6] S. Dang, O. Amin, B. Shihada and M.-S. Alouini, "What should 6G be?," Nature Electronics, vol. 3, pp. 20-29, 2020, doi: 10.1038/s41928-019-0355-6.

[7] E. C. Strinati, S. Barbarossa, J. L. Gonzalez-Jimenez, D. Kténas, N. Cassiau and C. Dehos, "6G: The next frontier," arXiv preprint arXiv:1901.03239, 2019.

[8] M. Latva-aho and K. Leppänen, "Key drivers and research challenges for $6 \mathrm{G}$ ubiquitous wireless intelligence," University of Oulu, While Paper, 2019.

[9] T. S. Rappaport et al., "Wireless communications and applications above $100 \mathrm{GHz}$ : Opportunities and challenges for 6G and beyond," IEEE Access, vol. 7, pp. 78729-78757, 2019, doi: 10.1109/ACCESS.2019.2921522.

[10] K. Nallappan, H. Guerboukha, C. Nerguizian and M. Skorobogatiy, "Uncompressed HD and Ultra-HD video streaming using terahertz wireless communications," in 2018 11th Global Symposium on Millimeter Waves (GSMM), 2018, pp. 1-3, doi: 10.1109/GSMM.2018.8439548.

[11] E. Basar, "Reconfigurable intelligent surface-based index modulation: A new beyond MIMO paradigm for 6G," IEEE Transactions on Communications, vol. 68, no. 5, pp. 3187-3196, May 2020, doi: 10.1109/TCOMM.2020.2971486.

[12] J. Wang et al., "Terabit free-space data transmission employing orbital angular momentum multiplexing," Nature photonics, vol. 6, pp. 488-496, 2012, doi: 10.1038/nphoton.2012.138.

[13] M. Giordani, M. Polese, M. Mezzavilla, S. Rangan and M. Zorzi, "Towards 6G networks: Use cases and technologies," arXiv preprint arXiv:1903.12216, 2019.

[14] M. Bennis, M. Debbah and H. V. Poor, "Ultrareliable and low-latency wireless communication: Tail, risk, and scale," Proceedings of the IEEE, vol. 106, no. 10, pp. 1834-1853, 2018, doi: 10.1109/JPROC.2018.2867029.

[15] S. Elmeadawy and R. M. Shubair, "Enabling technologies for 6G future wireless communications: opportunities and challenges," arXiv preprint arXiv:2002.06068, 2020. 
[16] K. B. Letaief, W. Chen, Y. Shi, J. Zhang and Y.-J. A. Zhang, "The roadmap to 6G: AI empowered wireless networks," IEEE Communications Magazine, vol. 57, no. 8, pp. 84-90, 2019, doi: 10.1109/MCOM.2019.1900271.

[17] W. Saad, M. Bennis and M. Chen, "A vision of 6G wireless systems: Applications, trends, technologies, and open research problems," IEEE network, vol. 34, no. 3, pp. 134-142, 2019, doi: 10.1109/MNET.001.1900287.

[18] H. Elayan, O. Amin, B. Shihada, R. M. Shubair and M.-S. Alouini, "Terahertz band: The last piece of RF spectrum puzzle for communication systems," IEEE Open Journal of the Communications Society, vol. 1, pp. 1-32, 2019, doi: 10.1109/OJCOMS.2019.2953633.

[19] N. Van Huynh, D. T. Hoang, X. Lu, D. Niyato, P. Wang and D. I. Kim, "Ambient backscatter communications: A contemporary survey," IEEE Communications Surveys \& Tutorials, vol. 20, no. 4, pp. 2889-2922, 2018, doi: 10.1109/COMST.2018.2841964.

[20] N. Qi, M. Xiao, T. A. Tsiftsis, M. Skoglund, P. L. Cao and L. Li, "Energy-efficient cooperative network coding with joint relay scheduling and power allocation," IEEE Transactions on Communications, vol. 64, no. 11, pp. 4506-4519, 2016, doi: 10.1109/TCOMM.2016.2602830.

[21] L. Su, C. Yang and I. Chih-Lin, "Energy and spectral efficient frequency reuse of ultra dense networks," IEEE Transactions on Wireless Communications, vol. 15, no. 8, pp. 5384-5398, 2016, doi: 10.1109/TWC.2016.2557790.

[22] D. Niyato, D. I. Kim, M. Maso and Z. Han, "Wireless powered communication networks: Research directions and technological approaches," IEEE Wireless Communications, vol. 24, no. 6, pp. 88-97, 2017, doi: 10.1109/MWC.2017.1600116.

[23] Y. Luo, L. Pu, G. Wang and Y. Zhao, "RF energy harvesting wireless communications: RF environment, device hardware and practical issues," Sensors, vol. 19, no. 13, p. 3010, 2019, doi: 10.3390/s19133010.

[24] S. Ulukus et al., "Energy harvesting wireless communications: A review of recent advances," IEEE Journal on Selected Areas in Communications, vol. 33, no. 3, pp. 360-381, 2015, doi: 10.1109/JSAC.2015.2391531.

[25] M. H. Alsharif, S. Kim and N. Kuruoğlu, "Energy harvesting techniques for wireless sensor networks/radiofrequency identification: a review," Symmetry, vol. 11, p. 865, 2019, doi: 10.3390/sym11070865.

\section{BIOGRAPHIES OF AUTHORS}
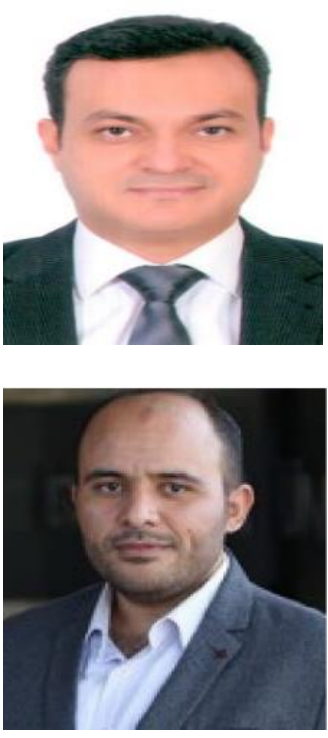

Ahmad A. A. Solyman received the degree from the University of Strathclyde, U.K., in 2013. His Ph.D. researches include in multimedia services over wireless networks using OFDM. He is currently an Assistant Professor with the Department of Electrical and Electronics Engineering, Istanbul Gelisim University, Turkey. His research interests contain wireless communication networks and MIMO communication systems.

Khalid Yahya received the Ph.D. degree in electrical engineering from Kocaeli University, Kocaeli, Turkey, in 2018. He is currently working as an Assistant Professor of mechatronics engineering with Istanbul Gelisim University, Turkey. He has published over a dozen articles in prestigious journals and conferences. He is an active reviewer of many conferences and journals. His current research interests include microelectronic circuit analysis and design, renewable energy resources, power electronics, and MPPT designs for energy harvesting systems and information security. 